\title{
Towards Building Wind Tunnels for Data Center Design
}

\author{
Avrilia Floratou \\ IBM Almaden \\ Research Center \\ aflorat@us.ibm.com
}

\author{
Frank Bertsch \\ University of \\ Wisconsin-Madison \\ bertsch@cs.wisc.edu
}

\author{
Jignesh M. Patel \\ University of \\ Wisconsin-Madison \\ jignesh@cs.wisc.edu
}

\author{
Georgios Laskaris \\ Duke \\ University \\ georgios.laskaris@duke.edu
}

\begin{abstract}
Data center design is a tedious and expensive process. Recently, this process has become even more challenging as users of cloud services expect to have guaranteed levels of availability, durability and performance. A new challenge for the service providers is to find the most cost-effective data center design and configuration that will accommodate the users' expectations, on ever-changing workloads, and constantly evolving hardware and software components. In this paper, we argue that data center design should become a systematic process. First, it should be done using an integrated approach that takes into account both the hardware and the software interdependencies, and their impact on users' expectations. Second, it should be performed in a "wind tunnel", which uses large-scale simulation to systematically explore the impact of a data center configuration on both the users' and the service providers' requirements. We believe that this is the first step towards systematic data center design - an exciting area for future research.
\end{abstract}

\section{INTRODUCTION}

Data centers are changing fast. Users of data centers running cloud applications are becoming increasingly demanding. These users now expect to have access to specific hardware resources (IOPs, memory capacity, number of CPU cores, network bandwidth), demand data availability and durability guarantees defined quantitatively in ServiceLevel-Agreements (SLAs) [1,3], and expect to get concrete performance guarantees defined in performance-based SLAs (e.g. $[12,13])$. Failure to satisfy these user requirements directly translates into financial loss for the data center/cloud provider. A natural question to ask in this situation is: "How should we design the data centers of the future?" In such settings, the data center provider wants to ask various "What if" questions, such as what is the cost vs. SLA implication of choosing one type of hard disk over the other, or using flash over hard disk storage, or adding more main memory to each node, or switching to a faster network card.

This work is licensed under the Creative Commons AttributionNonCommercial-NoDerivs 3.0 Unported License. To view a copy of this license, visit http://creativecommons.org/licenses/by-nc-nd/3.0/. Obtain permission prior to any use beyond those covered by the license. Contact copyright holder by emailing info@vldb.org. Articles from this volume were invited to present their results at the 40th International Conference on Very Large Data Bases, September 1st - 5th 2014, Hangzhou, China.

Proceedings of the VLDB Endowment, Vol. 7, No. 9

Copyright 2014 VLDB Endowment 2150-8097/14/05.
Typically, data center designers determine the hardware setup based on the software that will be deployed, and the expected workload characteristics. For example, in an environment where high availability is important, the data is replicated. The value for the replication factor $n$ is typically set to a "reasonable" value (e.g. 3 or 5), and storage is provisioned to accommodate these $n$ copies. However, it is not clear if this approach is the most cost-effective solution that provides the desired availability level. In some environments, one can reduce the replication factor to $n-1$, thereby decreasing the storage cost and increasing the speed of the repair process that re-replicates data in case of a hardware failure. Furthermore, the latency of the repair process can be reduced by using a faster network (hardware), or by optimizing the repair algorithm (software), or both. For example, by instantiating parallel repairs on different machines, one can decrease the probability that the data will become "unavailable" (i.e. the system has zero up-to-date copies of the data). Even if this solution may potentially provide lower availability than n-way replication (depending on the characteristics of the network or the repair algorithm), the resulting availability level may still satisfy the SLA. As we will show in the following sections, similar observations can be made for other design choices too, like data placement algorithms or replication protocols. These observations suggest that an iterative approach to data center design, which determines the software configuration first and then the hardware setup (or vice versa), may not be always "optimal" since it ignores important interactions between these two components. What we need is an integrated approach that explores the hardware/software interdependencies during the data center design process.

In this paper, we discuss the following three methods for integrated data center design: (a) perform what-if analyses on an actual scaled-down prototype of the data center, (b) use purely analytical methods to model the data center, and (c) use a "wind tunnel" to simulate the entire spectrum of hardware/software co-design space for data center design (and incremental re-design/upgrade). We show that the first two approaches have limitations that the third one avoids.

Thus, we envision the creation of a simulation-based data center wind tunnel that can be used to explore, experiment, and compare design choices, and to statistically reason about the guarantees that these design choices can provide.

\section{INTEGRATED DATA CENTER DESIGN}

In this section we discuss three approaches to explore hardware-software co-design for data center (DC) design. 


\subsection{What-if Analysis on a Small Data Center}

One approach is to actually experiment with different types of configurations on a small cluster, and perform whatif analyses on the prototype cluster. This approach has two key drawbacks. First, it is expensive to carry out such analyses to explore a large configuration space, both from the financial perspective and from the perspective of the time required to explore the configuration space. Second, it is challenging to pick the "correct" prototype cluster size as some behaviors that happen at a larger scale can't be easily observed at a smaller scale; e.g., increased communication latency or correlated hardware failures in an large scale cluster are harder to re-produce in a smaller prototype cluster.

We acknowledge that validating results from a simulator is challenging, and an actual smaller scale prototype can help with ensuring that the simulator works at least on the smaller scale. The smaller scale prototype can also help identify factors that the simulation must consider carefully.

\subsection{Analytical Modeling}

Analytical modeling has been widely used to study the behavior of complex systems (e.g., [6]), but such models may result in poor accuracy for the following reasons.

First, the (popular) M/M/1 and M/M/c models assume that the times to failure and repair are exponentially distributed. However, real distributions are not always exponential. For example, hard disk drive failure distribution typically follows the Weibull or Gamma distribution [15]. Repair times have also been shown to follow the lognormal distribution [16]. The more complex G/G/1 and G/G/C models, which capture more general distributions don't have a closed-form solution. While these more complex models can be approximated, as discussed in previous work [11], their accuracy is often inadequate.

Second, it is hard to capture all the parameters in the design space with an analytical model. For example, capturing the failure behavior of both the storage and the network layer as well as the repair behavior of the system, using a Markov Model is challenging. Typically the failure behavior of the network components (NIC cards, network switches) is ignored to in order to make the problem tractable. Capturing such complex behaviors accurately with an analytical model is challenging. Capturing the combination of each and every behavior in the design space is even more challenging.

Finally, using analytical models requires deep understanding of the related mathematical concepts, which are not widespread in practice, making it challenging to use this approach in practice. To fix this problem, we hope that more schools will offer courses in quantitative systems modeling sadly it appears that there are fewer schools that offer these courses in CS departments than a decade ago. We note that analytical models are crucial to better understanding and validating simulation models before the simulator can be used to answer a broader class of what-if questions, and we advocate using analytical models in that role.

\subsection{Simulation-based Wind Tunnel}

Our proposal to tackle the problem of integrated data center design, is to make use of a simulation-based "wind tunnel". We believe that this is the first step towards systematic data center design - a rich area of research with potentially high-impact on our industry.
In a wind tunnel the behavior of all the components (hardware, software) under consideration is simulated, with the goal of exploring the behavior of a more complex model. Note that in this case, we are not restricted to exponential distributions as in many analytical models, but we can incorporate any type of distribution.

The simulation-based wind tunnel avoids the hassle of purchasing and repetitively deploying the hardware and software, until we explore all the possible design ideas. Thus, it is a more economical way to investigate DC design choices than using a "sample" DC. At the same time is not restricted, by the size of the "sample" DC.

We note that this philosophy already has some proponents, though in a narrow domain (e.g., [11] for hardware, and $[9,18]$ for Hadoop parameter selection). What is missing is a general and extensible method that provides a comprehensive framework to evaluate the combination of both hardware (storage, CPU, networking and memory components) and software (e.g., replication policy, changes in network priority schemes in SDNs, parameter tuning) design choices. Such a framework is essential to allow holistic and comprehensive evaluation of the entire space of data center design alternatives for end-to-end what-if analyses.

\section{WIND TUNNEL USE CASES}

In this section we discuss some of the potential DC design problems that the wind tunnel can tackle.

Performance SLAs: To satisfy performance SLAs, workload characterization is needed in order to quantify the impact on existing workloads when a new workload is added on a machine. Existing work in prediction modeling for certain classes of database workloads [12], has shown that it is possible to build accurate models for a given DBMS by identifying and carefully modeling the key characteristics (e.g., CPU, Disk I/O, network, etc.) of the system under test. This observation, encourages us to believe that simulation can be used as a "cheap" way to explore interactions between workloads as long as the key resources are simulated. Our plan is to investigate how much detail the models must capture in order to produce accurate workload predictions and to also validate the predictions on real workloads/environments.

To the best of our knowledge, a performance prediction method that takes into account the impact of other cluster events (e.g., hardware failures, control operations) on workload performance, has not been proposed. Carefully designed, holistic simulation (aka. the "wind tunnel" that we propose) can capture the impact of these events on the performance SLAs, and result in more realistic predictions.

Availability SLAs: Data availability depends on both the hardware (e.g. hardware failure rate) and the software (e.g. replication, erasure codes [14]) characteristics. The wind tunnel is an extensible environment in which various software and hardware system components can be simulated together. Thus, it can be used to experiment with various configurations and collect insights about the impact of each design choice on the availability SLAs. See Section 4 for the challenges associated with modeling these components.

Hardware provisioning: Service providers need to ask questions such as: "Should I invest in storage or memory in order to satisfy the SLAs of $95 \%$ of my customers and minimize the total operating cost?" These questions can be described as queries that the wind tunnel can help answer. 


\section{RESEARCH CHALLENGES}

We believe that building a wind tunnel is similar to building a specialized data processing system. Thus, the database community is well poised to address the problem of combining models and data in a wind tunnel for DC design. Queries to the wind tunnel are design questions that iterate over a vast design space of DC configurations. In this section we discuss the associated research challenges.

\subsection{Declarative Simulation Processing}

Database researchers have already highlighted the benefits of declarative query processing for computer games [19] and network services [10]. We believe that declarative methods can also help in this setting to capture model interactions and end-user/SLA requirements.

Model interactions: When a new model is added to the simulator, its interactions with the existing models should be declaratively specified. For example, a model that simulates a data transfer to a simulated machine is not independent of a model that simulates a workload executed on that machine as both models impact each other when they happen to be invoked on the same machine in the simulation. On the other hand, the failure model of the hard disk is independent of the failure model of the network switch. The underlying simulation engine can then automatically optimize and parallelize the query execution based on the user's declarations. Capturing the model interactions declaratively can lead to a modular and extensible wind tunnel design.

Expressing the desired DC constraints: The design questions can often be complicated: for example, the user may need to specify a required SLA as a distribution. Posing such queries declaratively is likely to be preferred over more imperative styles of querying.

We believe, that exploring the design and implementation of declarative languages to support the two aspects discussed above is a valuable area for future research. Perhaps we could build such a language based on existing declarative languages like Datalog or SQL.

\subsection{Simulation at Scale}

Simulating multiple components of a DC is likely to be time-consuming. We may need to borrow techniques used to scale database queries (query optimization, parallelization) and apply them in the context of the wind tunnel execution. One approach is to take the following two-step process.

In the first step (optimization), an order for the simulation runs is specified that would facilitate dismissing certain simulation runs by simply observing the output of the previous runs. For example, if a performance SLA cannot be met with a $10 \mathrm{~Gb}$ network, then it won't be met with a $1 \mathrm{~Gb}$ network, while all other design parameters remain the same. Thus, the simulation run with the $10 \mathrm{~Gb}$ configuration should precede the run with the $1 \mathrm{~Gb}$ configuration. Extending this idea to more than one dimension is an interesting research problem.

In the following step (parallelization), each simulation run is parallelized based on the model interactions (perhaps specified declaratively as discussed above). For example, the affected components during the completion of a data transfer from one node in a rack to another node in the same rack are: the two nodes, the two disks where the data was streaming to and from, and the switch itself. Work done on other nodes within the rack is unaffected, and work done between any nodes not in the rack remains unaffected as well. Parallel execution of such events provides an easy way to scale the simulator. Although, the problem of parallelizing simulations has been studied before (e.g., [7]), declarative query processing creates new opportunities and challenges. The logic specific to the models being simulated must now be captured when declaring the models and not in the simulation engine itself. As a result, the simulation engine should be designed to gracefully adjust to various user declarations. Finding ways to abstract the existing simulation parallelization techniques to allow coupling with a declarative specification language is an interesting area for research.

Another approach to speed up execution, is to monitor the simulation progress and abort a simulation run before it completes, if it is clear from the existing progress that the design constraint (e.g., a desired SLA) will not be met. Exploiting this knowledge to refine the order of future configurations is another interesting problem, and potentially similar to dynamic query optimization.

\subsection{Validating the Simulator}

Another challenge is how to validate the simulator. Simple simulation models can be validated using analytical models. The predictions of crucial models can be tested on a small actual hardware setup, whenever that is possible. Another way to validate the simulation results is to use publicly available datasets that describe the behavior of real clusters. There are a few datasets of this kind (e.g., [15]), but we certainly need more data of this nature.

In fact, one hope that we have is that a broader recognition of this area of systematic DC design will spur researchers to invest the time that is needed to produce datasets from actual deployments, which can then be used in wind tunnel simulations. Perhaps, projects like OpenCompute [2], could expand from their current charter to take on the challenge of making data from large operational systems available on a continual basis, especially for newer hardware components. It would also be useful to get sanitized hardware and software logs from actual clusters to validate selected simulations (e.g., response time predictions).

\subsection{Managing the Wind Tunnel Data}

The data related to the wind tunnel falls into two categories: (a) data generated by wind tunnel simulations, and (b) operational log data from DCs used to build data-driven models for the simulator components.

Akin to the situation with simulation data in the sciences, we expect that a large amount of simulation data (generated by the process of exploring large portions of the DC design space) will be collected over time. This data can be subjected to deep exploratory analysis. For example, users may question whether they have already explored a configuration scenario "similar" to a target scenario, or question what is a typical pattern of failures for a specific configuration. Determining how to organize the output of the simulation along with the input model data (e.g., should we use a relational store or a schema-less key-value store?) to facilitate these kinds of analyses is an open problem. The varied nature of this data makes this problem challenging $[4,8]$.

Identifying how to store and process operational log data from real DCs in order to seed data-driven models (e.g., for an SSD device type) is an interesting problem. As mentioned in [8], transformation algorithms that convert log data into 


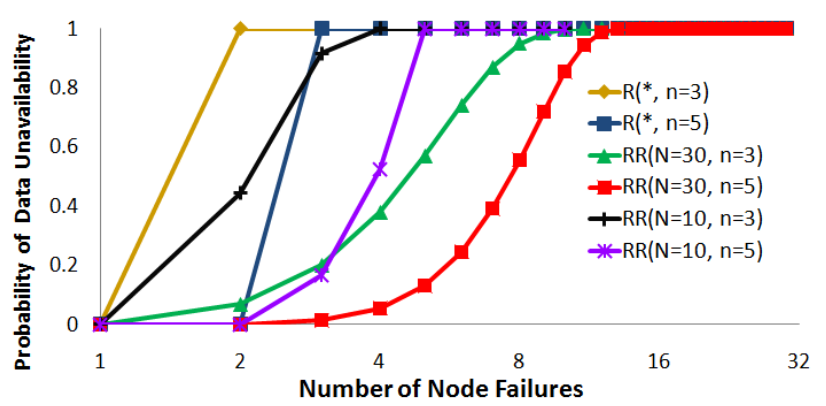

Figure 1: Probability of data unavailability

meaningful models (e.g., probability distributions) that can be used by the wind tunnel, must be developed. The related data privacy issues must also be addressed.

\subsection{Modeling the Hardware Components}

Satisfying availability and durability SLAs, requires precise knowledge of the failure characteristics of each hardware component. For example, modeling the failure behavior of a hard disk requires a good estimate of the distribution that time between disk replacements follows. Although hardware manufacturer's typically enclose the values of the mean time to failure and the annualized failure rate, these numbers do not necessarily reflect the actual disk behavior in practice. In fact, these numbers tend to overestimate the hardware capabilities [15]. Over the last few years, more hardware failure studies have been conducted [16,17], but the entire space of hardware components (such as I/O controllers, memory modules, etc), has still not been covered.

Another problem often encountered in large DCs is hardware whose performance deteriorates significantly compared to its specification [5]. This kind of behavior (e.g., an underperforming NIC card) is hard to reproduce in practice. Examining how to model these "failures" across the hardware components spectrum is an exciting area of future research.

\subsection{Modeling the Software Components}

Besides better hardware models, we also need models for software components. One key challenge in modeling software is to decide which software design choices affect the SLA requirements, and as a result should be simulated.

As an example, we present early results from a simulator that we are building. Here we simulate a cloud setting in which data is replicated for high availability. Figure 1 shows the probability of having at least one customer's data become unavailable as the number of node failures in the cluster increases, for varying cluster sizes, data placement algorithms and replication factors. We assume that the service uses a quorum-based protocol. In this case, if the majority of data replicas of a given customer are unavailable, then the customer is not able to operate on the data. We use the Random ( $R$ ) and Round Robin ( $R R$ ) data placement policies to distribute the replicas across the machines. We use 10,000 users, two replication factors $(n=3, n=5)$ and two configurations ( $\mathrm{N}=10$ and $\mathrm{N}=30$ nodes $)$. The * symbol denotes both cluster configurations. As the figure shows, the probability of data unavailability varies across different configurations. It depends on the cluster size, the replication factor and the data placement policy. Thus, all of these parameters must be captured in the simulation in order to understand the system's availability behavior.
Determining which software design choices interact, and as a whole affect a specific aspect of the system's behavior, such as data availability, is challenging. Failure to model a critical parameter may result in inaccurate simulation results. We need to systematically analyze existing complex software systems and determine which parameters are independent from the others and which ones interact with others. Although this categorization is needed for accurate DC design with the wind tunnel, the categorization can be made easier by making use of the wind tunnel itself! This is because the wind tunnel constitutes a framework in which we can add or remove properties and get insights on the effect that these produce at a global scale - thus the wind tunnel can help software engineers make better design choices.

\section{CONCLUSIONS}

It is too expensive and sub-optimal to design data centers using existing methods. As the diversity of the hardware components ecosystem (e.g., new storage device types, low powered processors, network speeds) continues to increase, along with an explosion in software methods (e.g., replication, data partitioning, distributed query processing), it is critical that we investigate simulation-based methods to answer holistic "what-if" questions on simulated data center designs. We proposed building a wind tunnel to address this problem, and outline various research challenges that must be addressed to realize this vision.

\section{ACKNOWLEDGMENTS}

This research was supported in part by a grant from the Microsoft Jim Gray Systems Lab (GSL), and by the National Science Foundation under grants IIS-0963993 and IIS1250886. Part of this work by A. Floratou was done while she was at the University of Wisconsin and at the GSL.

\section{7.}

[2] Open compute project http://wn

[3] Windows azure slas. http://www. windowsazure.com/en-us/support/legal/sla/.

[4] P. A. Bernstein, A. Y. Halevy, and R. A. Pottinger. A vision for

management of complex models. SIGMOD Rec., 29(4):55-63, Dec. 2000.

[5] T. Do, M. Hao, T. Leesatapornwongsa, T. Patana-anake, and H. S. Gunawi. Limplock: Understanding the impact of limpware on scale-out cloud systems. In SOCC, 2013.

[6] D. Ford, F. Labelle, F. I. Popovici, M. Stokely, V.-A. Truong, L. Barroso, C. Grimes, and S. Quinlan. Availability in globally distributed storage $-74,2010$

[7] R. Fujimoto. Parallel discrete event simulation. Commun. ACM $33(10): 30-53,1990$

[8] P. J. Haas, P. P. Maglio, P. G. Selinger, and W. C. Tan. Data is dead... without what-if models. PVLDB, 4(12):1486-1489, 2011

[9] H. Herodotou and S. Babu. A what-if engine for cost-based mapreduce optimization. IEEE Data Eng. Bull., 36(1):5-14, 2013.

[10] B. T. Loo, T. Condie, M. Garofalakis, D. E. Gay, J. M. Hellerstein, P. Maniatis, R. Ramakrishnan, T. Roscoe, and I. Stoica. Declarative networking. Commun. ACM, 52(11):87-95, Nov. 2009

[11] D. Meisner, J. Wu, and T. F. Wenisch. Bighouse: A simulation infrastructure for data center systems. In ISPASS, pages $35-45,2012$

[12] B. Mozafari, C. Curino, and S. Madden. Dbseer: Resource and performance prediction for building a next generation database cloud. In $C I D R, 2013$.

[13] V. R. Narasayya, S. Das, M. Syamala, B. Chandramouli, and S. Chaudhuri. Sqlvm: Performance isolation in multi-tenant relational database-as-a-service. In CIDR, 2013

[14] M. Sathiamoorthy, M. Asteris, D. Papailiopoulos, A. G. Dimakis, R. Vadali, S. Chen, and D. Borthakur. Xoring elephants: Novel erasure codes for big data. Proc. VLDB Endow., 6(5):325-336, Mar. 2013.

[15] B. Schroeder and G. A. Gibson. Disk failures in the real world: What does an mttf of $1,000,000$ hours mean to you? In FAST, pages 1-16, 2007.

[16] B. Schroeder and G. A. Gibson. A large-scale study of failures in high-performance computing systems. IEEE Trans. Dependable Sec. Comput., $7(4): 337-351,2010$

[17] K. V. Vishwanath and N. Nagappan. Characterizing cloud computing hardware reliability. In SoCC, pages 193-204, 2010.

[18] G. Wang, A. R. Butt, P. Pandey, and K. Gupta. A simulation approach to evaluating design decisions in mapreduce setups. In MASCOTS, pages 1-11, 2009 .

[19] W. White, C. Koch, N. Gupta, J. Gehrke, and A. Demers. Database research opportunities in computer games. SIGMOD Rec., 36(3):7-13, 2007. 